\title{
COEFFICIENT PROBLEMS ON THE CLASS $U(\lambda)$
}

Abstract. For $0<\lambda \leq 1$, let $\mathcal{U}(\lambda)$ denote the family of functions $f(z)=z+\sum_{n=2}^{\infty} a_{n} z^{n}$ analytic in the unit disk $\mathbb{D}$ satisfying the condition $\left|\left(\frac{z}{f(z)}\right)^{2} f^{\prime}(z)-1\right|<\lambda$ in $\mathbb{D}$. Although functions in this family are known to be univalent in $\mathbb{D}$, the coefficient conjecture about $a_{n}$ for $n \geq 5$ remains an open problem. In this article, we shall first present a non-sharp bound for $\left|a_{n}\right|$. Some members of the family $\mathcal{U}(\lambda)$ are given by

$$
\frac{z}{f(z)}=1-(1+\lambda) \phi(z)+\lambda(\phi(z))^{2}
$$

with $\phi(z)=e^{i \theta} z$, that solve many extremal problems in $\mathcal{U}(\lambda)$. Secondly, we shall consider the following question: Do there exist functions $\phi$ analytic in $\mathbb{D}$ with $|\phi(z)|<1$ that are not of the form $\phi(z)=e^{i \theta} z$ for which the corresponding functions $f$ of the above form are members of the family $\mathcal{U}(\lambda)$ ? Finally, we shall solve the second coefficient $\left(a_{2}\right)$ problem in an explicit form for $f \in \mathcal{U}(\lambda)$ of the form

$$
f(z)=\frac{z}{1-a_{2} z+\lambda z \int_{0}^{z} \omega(t) d t},
$$

where $\omega$ is analytic in $\mathbb{D}$ such that $|\omega(z)| \leq 1$ and $\omega(0)=a$, where $a \in \overline{\mathbb{D}}$.

Key words: Univalent function, subordination, Julia's lemma, Schwarz' lemma

\section{Mathematical Subject Classification: $30 C 45$}

We denote the unit disk by $\mathbb{D}=\{z \in \mathbb{C}:|z|<1\}$, and let $\mathcal{H}$ be the linear space of analytic functions defined on $\mathbb{D}$ endowed with the topology 
of locally uniform convergence and $\mathcal{A}=\left\{f \in \mathcal{H}: f(0)=f^{\prime}(0)-1=0\right\}$. The family $\mathcal{S}$ of univalent functions from $\mathcal{A}$ and many of its subfamilies, for which the image domains have special geometric properties, have been investigated in detail. Among them are convex, starlike, close-to-convex, spirallike and typically real mappings. For the general theory of univalent functions we refer the reader to the books [7, 10, 23]. The class $\mathcal{U}(\lambda)$ defined below seems to have many interesting properties (cf. [21, 22]). For $0<\lambda \leq 1$, we consider the family

$$
\mathcal{U}(\lambda)=\left\{f \in \mathcal{A}:\left|U_{f}(z)\right|<\lambda \text { in } \mathbb{D}\right\},
$$

where

$$
U_{f}(z)=\left(\frac{z}{f(z)}\right)^{2} f^{\prime}(z)-1=\frac{z}{f(z)}-z\left(\frac{z}{f(z)}\right)^{\prime}-1, \quad z \in \mathbb{D} .
$$

Set $\mathcal{U}:=\mathcal{U}(1)$, and observe that $\mathcal{U} \subsetneq \mathcal{S}($ see $[1,2])$.

Before we continue the discussion, it might be appropriate to include a few well-known properties about the family $\mathcal{U}(\lambda)$.

1) Let $\Sigma$ denote the family of univalent functions $F$ of the form,

$$
F(\zeta)=\zeta+\sum_{n=0}^{\infty} c_{n} \zeta^{-n}, \quad|\zeta|>1,
$$

which satisfies the condition $F(\zeta) \neq 0$ for $|\zeta|>1$. Then we observe that each $f \in \mathcal{S}$ can be associated with a mapping $F \in \Sigma$ by the correspondence

$$
F(\zeta)=\frac{1}{f(1 / \zeta)}, \quad|\zeta|>1
$$

Using the change of variable $\zeta=\frac{1}{z}$, the association $f(z)=1 / F\left(\frac{1}{z}\right)$ quickly yields the formula

$$
F^{\prime}(\zeta)-1=U_{f}(z)
$$

and

$$
\frac{z f^{\prime}(z)}{f(z)}=\frac{\zeta F^{\prime}(\zeta)}{F(\zeta)}
$$

where $U_{f}$ is defined by (1). Consequently, for $0<\lambda \leq 1$, the formula (2) gives that $f \in \mathcal{U}(\lambda)$ if and only if $\left|F^{\prime}(\zeta)-1\right|<\lambda$ for $|\zeta|>1$. The formula (3) could be used to connect the starlikeness of $f$ with that of $F$. 
2) An interesting fact is that each function in

$$
\mathcal{S}_{\mathbb{Z}}=\left\{z, \frac{z}{(1 \pm z)^{2}}, \frac{z}{1 \pm z}, \quad \frac{z}{1 \pm z^{2}}, \frac{z}{1 \pm z+z^{2}}\right\}
$$

belongs to $\mathcal{U} \cap \mathcal{S}^{*}$, where $\mathcal{S}^{*}$ denoted the family of starlike functions $f$ on $\mathbb{D}$, i.e., univalent functions $f$ such that $f(\mathbb{D})$ is a domain starlike with respect to the origin. Also, it is well-known that these are the only functions in $\mathcal{S}$ having integer coefficients in the power series expansions of functions $f \in \mathcal{S}$ (see [9]).

3) The family $\mathcal{U}$ is not a subset of the starlike family $\mathcal{S}^{*}$ as the function

$$
f_{1}(z)=\frac{z}{1+\frac{1}{2} z+\frac{1}{2} z^{3}}
$$

demonstrates. Indeed, it is easy to see that $f_{1} \in \mathcal{U}$ and

$$
\frac{z f_{1}^{\prime}(z)}{f_{1}(z)}=\frac{1-z^{3}}{1+\frac{1}{2} z+\frac{1}{2} z^{3}}
$$

and at $z_{0}=(-1+i) / \sqrt{2},\left|z_{0}\right|=1$, we obtain that

$$
\operatorname{Re}\left\{\frac{z_{0} f_{1}^{\prime}\left(z_{0}\right)}{f_{1}\left(z_{0}\right)}\right\}=\frac{2-2 \sqrt{2}}{3}<0,
$$

from which it follows that the function $f_{1}$ is not starlike in $\mathbb{D}$. See also [16].

4) One of the sufficient conditions for a function $f(z)=z+\sum_{n=2}^{\infty} a_{n} z^{n}$ to be in $\mathcal{S}^{*}$ is that $\sum_{n=2}^{\infty} n\left|a_{n}\right| \leq 1$ and this result is sharp. In spite of the fact that neither $\mathcal{S}^{*}$ is included in $\mathcal{U}$ nor includes $\mathcal{U}$, it is known that (see also [8]) the condition $\sum_{n=2}^{\infty} n\left|a_{n}\right| \leq 1$ implies that $f \in \mathcal{U}$. The result is sharp. On the other hand, if $f(z)=z-\sum_{n=2}^{\infty}\left|a_{n}\right| z^{n}$ is in $\mathcal{S}^{*}$, then $f \in \mathcal{U}$. See [17.

5) Since $f(z)=z-\sum_{n=2}^{\infty}\left|a_{n}\right| z^{n}$ is in $\mathcal{S}^{*}$ if and only if $\sum_{n=2}^{\infty} n\left|a_{n}\right| \leq 1$ (see [25, Theorem 2]), this result can be used to generate univalent functions $f \in \mathcal{U}$ that are not starlike. 
6) Since functions in $\mathcal{S}$ are not necessarily in $\mathcal{U}$, it is natural to consider the largest value $r_{0}$ so that for each $f \in \mathcal{S}$ the function $\frac{1}{r} f(r z) \in \mathcal{U}$ for $0<r \leq r_{0}$. In this case we say that $r_{0}:=r_{\mathcal{U}}(\mathcal{S})$ is the $\mathcal{U}$ radius (or the radius of $\mathcal{U}$-property) in the class $\mathcal{S}$. It is known that ([15]), $r_{\mathcal{U}}(\mathcal{S})=\frac{1}{\sqrt{2}}$. More generally, $r_{\mathcal{U}(\lambda)}(\mathcal{S})=\sqrt{\frac{\lambda}{1+\lambda}}$. There has been a long history in determining radii problems in the theory of univalent functions, see [10].

7) In [18, it was shown that if $f \in \mathcal{U}(\lambda), a:=\left|f^{\prime \prime}(0)\right| / 2 \leq 1$ and $0 \leq \lambda \leq \frac{\sqrt{2-a^{2}}-a}{2}$, then $f \in \mathcal{S}^{*}$. Later Fournier and Ponnusamy [8] have proved that the upper bound on $\lambda$ is sharp. Moreover, they have shown that there exist non-starlike functions $f \in \mathcal{U}$ such that

$$
0<\frac{\sqrt{2-a^{2}}-a}{2}<\sup _{z \in \mathbb{D}}\left|f^{\prime}(z)\left(\frac{z}{f(z)}\right)^{2}-1\right| \leq 1-a .
$$

Recently, a number of useful properties of the family $\mathcal{U}(\lambda)$ were established in [19, 20, 21, 22]. However, the coefficient problem for $\mathcal{U}(\lambda)$ remains open. This article supplements the earlier investigations in this topic.

Let $\mathcal{B}=\{\omega \in \mathcal{H}:|\omega(z)|<1$ on $|z|<1\}$ and $\mathcal{B}_{0}=\{\omega \in \mathcal{B}: \omega(0)=0\}$. In addition, for $f, g \in \mathcal{H}$, we use the symbol $f(z) \prec g(z)$, or in short $f \prec g$, to mean that there exists an $\omega \in \mathcal{B}_{0}$ such that $f(z)=g(\omega(z))$. We now recall the following results from [19] which we need in the sequel.

Theorem A. Suppose that $f \in \mathcal{U}(\lambda)$ for some $\lambda \in(0,1]$ and $a_{2}=$ $=f^{\prime \prime}(0) / 2$. Then we have the following:

(a) If $\left|a_{2}\right|=1+\lambda$, then $f$ must be of the form

$$
f(z)=\frac{z}{\left(1+e^{i \theta} z\right)\left(1+\lambda e^{i \theta} z\right)} .
$$

(b) $\frac{z}{f(z)}+a_{2} z \prec 1+2 \lambda z+\lambda z^{2} \quad$ and $\quad \frac{f(z)}{z} \prec \frac{1}{(1-z)(1-\lambda z)}, z \in \mathbb{D}$.

As an analogue to the famous estimate for the Taylor coefficients of univalent functions proved by de Branges [5] (see also [3]), the following conjecture was proposed in [19. 
Conjecture 1. Suppose that $f \in \mathcal{U}(\lambda)$ for some $0<\lambda \leq 1$ and $f(z)=$ $=z+\sum_{n=2}^{\infty} a_{n} z^{n}$. Then $\left|a_{n}\right| \leq \sum_{k=0}^{n-1} \lambda^{k}$ for $n \geq 2$.

This conjecture has been verified for $n=2$ first in 26 and a simpler proof was given in [19. More recently, in [21], Obradović et al. proved the conjecture for $n=3,4$ with an alternate proof for the case $n=2$, but it remains open for all $n \geq 5$. Because $\mathcal{U}(1) \subsetneq \mathcal{S}$ and the Koebe function belongs to $\mathcal{U}(1)$, this conjecture obviously holds for $\lambda=1$, in view of the de Branges theorem. Since no bound has been obtained for $\left|a_{n}\right|$ for $n \geq 5$, it seems useful to obtain a reasonable estimate. This attempt gives the following theorem and at the same time the proof for the case $\lambda=1$ does not require the use of de Branges theorem that $\left|a_{n}\right| \leq n$ for $f \in \mathcal{S}$ with equality for the Koebe function and its rotations.

Theorem 1. Let $f(z)=z+\sum_{n=2}^{\infty} a_{n} z^{n}$ belong to $\mathcal{U}(\lambda)$ for some $0<\lambda \leq 1$. Then

$$
\left|a_{n}\right| \leq 1+\lambda \sqrt{n-1} \sqrt{\sum_{k=0}^{n-2} \lambda^{2 k}}, \text { for } n \geq 2 .
$$

Proof. Let $f \in \mathcal{U}(\lambda)$. Then the second subordination relation in Theorem $\mathrm{A}(\mathrm{b})$ shows that

$$
\frac{f(z)}{z} \prec \frac{1}{1-\lambda z} \frac{1}{1-z}=f_{1}(z) f_{2}(z), \quad z \in \mathbb{D} .
$$

This means that there exists a function $\phi \in \mathcal{B}_{0}$ such that

$$
\frac{f(z)}{z}=f_{1}(\phi(z)) f_{2}(\phi(z)), \quad z \in \mathbb{D} .
$$

Define $g_{1}(z)=f_{1}(\phi(z))$ and $g_{2}(z)=f_{2}(\phi(z))$. Then

$$
g_{1}(z)=\sum_{n=0}^{\infty} b_{n} z^{n} \prec f_{1}(z)=\frac{1}{1-\lambda z} \text { and } g_{2}(z)=\sum_{n=0}^{\infty} c_{n} z^{n} \prec f_{2}(z)=\frac{1}{1-z} \text {, }
$$

where $b_{0}=c_{0}=1$, Rogosinski's theorems [24] (see also [7, Theorems 6.2 and 6.4]) give that

$$
\sum_{k=1}^{n}\left|b_{k}\right|^{2} \leq \sum_{k=1}^{n} \lambda^{2 k} \text { and }\left|c_{n}\right| \leq 1 \text { for } n \geq 1 .
$$


Moreover, the relation $\frac{f(z)}{z}=g_{1}(z) g_{2}(z)$ gives

$$
a_{n+1}=\sum_{k=0}^{n} b_{k} c_{n-k}
$$

Consequently, by (4), it follows from the classical Cauchy-Schwarz inequality that

$$
\left|a_{n+1}\right| \leq 1+\sum_{k=1}^{n}\left|b_{k}\right| \leq 1+\sqrt{n} \sqrt{\sum_{k=1}^{n}\left|b_{k}\right|^{2}} \leq 1+\sqrt{n} \sqrt{\sum_{k=1}^{n} \lambda^{2 k}},
$$

which implies the desired assertion.

Suppose that $f \in \mathcal{U}(\lambda)$. Then the second subordination relation in Theorem $\mathrm{A}(\mathrm{b})$ shows that there exists a function $\phi \in \mathcal{B}_{0}$ such that

$$
\frac{z}{f(z)}=1-(1+\lambda) \phi(z)+\lambda(\phi(z))^{2}, \quad z \in \mathbb{D} .
$$

From Theorem $\mathrm{A}(\mathrm{a})$, we see that there is a member in the family $\mathcal{U}(\lambda)$ in the above form with $\phi(z)=e^{i \theta} z$. In this type of functions, we have $\left|a_{2}\right|=1+\lambda$. A natural question is whether there exist functions $\phi \in \mathcal{B}_{0}$ that are not of the form $\phi(z)=e^{i \theta} z$ of the above type for which the corresponding $f$ of the form (5) belongs to $\mathcal{U}(\lambda)$. In order to prove the next result, we need the classical Julia lemma which is often quoted as Jack's lemma [12, Lemma 1] or Clunie-Jack's lemma [6] although this fact was known much before the work of Jack. See the article of Boas [4] for a historical commentary.

Lemma B. Let $\left|z_{0}\right|<1$ and $r_{0}=\left|z_{0}\right|$. Let $f(z)=\sum_{k=n}^{\infty} a_{k} z^{k}$ be continuous on $|z| \leq r_{0}$ and analytic on $\left\{z:|z|<r_{0}\right\} \cup\left\{z_{0}\right\}$ with $f(z) \not \equiv 0$ and $n \geq 1$. If $\left|f\left(z_{0}\right)\right|=\max _{|z| \leq r_{0}}|f(z)|$, then $z_{0} f^{\prime}\left(z_{0}\right) / f\left(z_{0}\right)$ is real number and $z_{0} f^{\prime}\left(z_{0}\right) / f\left(z_{0}\right) \geq n$.

Theorem 2. Let $f \in \mathcal{U}(\lambda)$ be given by (5), with a function $\phi$ analytic on the closed unit disk $\overline{\mathbb{D}}$ and a point $e^{i \theta_{0}}$ such that $\phi\left(e^{i \theta_{0}}\right)=-1$. Then $\phi$ is of the form $\phi(z)=e^{i \theta} z$. 
Proof. We observe that $f \in \mathcal{U}(\lambda)$ if and only if

$$
\left|\frac{z}{f(z)}-z\left(\frac{z}{f(z)}\right)^{\prime}-1\right|<\lambda, \quad z \in \mathbb{D},
$$

which according to (1) and (5) implies that there exists a function $\phi \in \mathcal{B}_{0}$ such that

$$
L(\phi)(z)=\left|-(1+\lambda)\left(\phi(z)-z \phi^{\prime}(z)\right)+\lambda \phi(z)\left(\phi(z)-2 z \phi^{\prime}(z)\right)\right|<\lambda, \quad z \in \mathbb{D} .
$$

Let us consider now a function $\phi$ analytic in $\overline{\mathbb{D}}$ such that there exists $\theta_{0}$ with $\phi\left(e^{i \theta_{0}}\right)=-1$. Examples of such functions are the Blaschke products. Now, we let $\tilde{\phi}(z)=\phi(r z)$ for $r>1$ and sufficiently close to 1 such that $\tilde{\phi}$ is analytic in $\mathbb{D}$. If we apply Julia's lemma with $n=1$ to $\tilde{\phi}$ and $z_{0}=e^{i \theta_{0}} / r$, we see that

$$
\frac{z_{0} \tilde{\phi}^{\prime}\left(z_{0}\right)}{\tilde{\phi}\left(z_{0}\right)}=\frac{e^{i \theta_{0}} \phi^{\prime}\left(e^{i \theta_{0}}\right)}{\phi\left(e^{i \theta_{0}}\right)}=m\left(\theta_{0}\right) \geq 1 .
$$

If we let $\phi(z)=z \psi(z)$, then we see that $\psi(\mathbb{D}) \subset \overline{\mathbb{D}}$ and $\psi\left(e^{i \theta_{0}}\right)=-e^{-i \theta_{0}}$. Now, we assume that $m\left(\theta_{0}\right)=1$. Since

$$
\frac{z \phi^{\prime}(z)}{\phi(z)}=1+\frac{z \psi^{\prime}(z)}{\psi(z)}
$$

this means that $\psi^{\prime}\left(e^{i \theta_{0}}\right)=0$. If $\psi^{\prime}$ is not a constant, an angle with width less than $\pi$, sufficiently close to $\pi$ and vertex $e^{i \theta_{0}}$ would be mapped by $\psi$ onto an angle with width sufficiently close to $2 \pi$ or more and a vertex $-e^{-i \theta_{0}}$. This contradicts the fact that $\psi(\mathbb{D}) \subset \overline{\mathbb{D}}$. Hence, $m\left(\theta_{0}\right)>1$ or $\phi$ is of the form $\phi(z)=e^{i \theta} z$. From the above we get

$$
e^{i \theta_{0}} \phi^{\prime}\left(e^{i \theta_{0}}\right)=-m\left(\theta_{0}\right)
$$

and therefore,

$$
\begin{gathered}
L(\phi)\left(z_{0}\right)=\left|-(1+\lambda)\left(\phi\left(z_{0}\right)-z_{0} \phi^{\prime}\left(z_{0}\right)\right)+\lambda \phi\left(z_{0}\right)\left(\phi\left(z_{0}\right)-2 z_{0} \phi^{\prime}\left(z_{0}\right)\right)\right|= \\
=\lambda+(1+3 \lambda)\left(m\left(\theta_{0}\right)-1\right) .
\end{gathered}
$$

If $m\left(\theta_{0}\right)>1$, then $L(\phi)\left(z_{0}\right)>\lambda$. This contradicts (6) and hence, $\phi(z)=$ $=e^{i \theta} z$. The proof is complete.

In [19, Theorem 5], under a mild restriction on $f \in \mathcal{U}(\lambda)$, the region of variability of $a_{2}$ is established as in the following form. 
Theorem C. Let $f \in \mathcal{U}(\lambda)$ for some $0<\lambda \leq 1$, and such that

$$
\frac{z}{f(z)} \neq(1-\lambda)(1+z), z \in \mathbb{D}
$$

Then, we have

$$
\frac{z}{f(z)}-(1-\lambda) z \prec 1+2 \lambda z+\lambda z^{2}
$$

and the estimate $\left|a_{2}-(1-\lambda)\right| \leq 2 \lambda$ holds. In particular, $\left|a_{2}\right| \leq 1+\lambda$ and the estimate is sharp as the function $f_{\lambda}(z)=z /((1+\lambda z)(1+z))$ shows.

Certainly, it was not unnatural to raise the question whether the condition (7) is necessary for a function $f$ to belong to the family $\mathcal{U}(\lambda)$. This question was indeed raised in [19]. In the next result, we show that the condition (7) cannot be removed from Theorem C. Before we present the proof, it is worth recalling from [19] that if $f \in \mathcal{U}(\lambda)$, then for each $R \in(0,1)$, the function $f_{R}(z)=R^{-1} f(R z)$ also belongs to $\mathcal{U}(\lambda)$.

Theorem 3. Let $f(z)=z /((1-z)(1-\lambda z))$ and for a fixed $R \in(0,1)$, let $f_{R}(z)=R^{-1} f(R z)$. Then we have

(a) For $0<\lambda \leq 1 / 2$ there exists, for any $R \in(0,1)$, an $r \in(0,1)$ such that $F(R, r)=0$, where

$$
F(R, r)=\frac{r}{f_{R}(r)}-(1-\lambda)(1+r) .
$$

(b) For $1 / 2<\lambda<1$ there exists, for any

$$
1>R>\frac{1+\lambda-\sqrt{(1-\lambda)(1+7 \lambda)}}{2 \lambda},
$$

an $r \in(0,1)$ such that $F(R, r)=0$.

Proof. We consider $F(R, r)$ given by $(9)$ and observe that

$$
F(R, r)=\lambda R^{2} r^{2}-r[R(1+\lambda)+1-\lambda]+\lambda .
$$

We see that in the cases indicated in the statement of the theorem $F(R, 0)=\lambda>0$ and $F(R, 1)<0$. Indeed

$$
F(R, 1)=\lambda R^{2}-R(1+\lambda)+2 \lambda-1=-R[(1-R) \lambda+1]-(1-2 \lambda)
$$


which is less than zero for any $R \in(0,1)$ and for $0<\lambda \leq 1 / 2$. Similarly, for the case $1 / 2<\lambda<1$, one can compute the roots of the equation $F(R, 1)=0$ and obtain the desired conclusion. This proves the assertion of Theorem 3 ,

Because of the characterization of functions in $\mathcal{U}(\lambda)$ via functions in $\mathcal{B}$, the following result is of independent interest. As pointed out in the introduction, it is known that if $f \in \mathcal{U}(\lambda)$, then $\left|a_{2}\right| \leq 1+\lambda$ with equality for $f(z)=z /[(1-z)(1-\lambda z)]$ and its rotations.

Theorem 4. Let $f \in \mathcal{U}(\lambda), \lambda \in(0,1)$, have the form

$$
f(z)=z+\sum_{n=2}^{\infty} a_{n} z^{n}=\frac{z}{1-a_{2} z+\lambda z \int_{0}^{z} \omega(t) d t}
$$

for some $\omega \in \mathcal{B}$ such that $\omega(0)=a \in \mathbb{D}$ and $v(x)$ be defined by

$$
v(x)=\int_{0}^{1} \frac{x+t}{1+x t} d t=\frac{1}{x}-\frac{1-x^{2}}{x^{2}} \log (1+x)<1 \text { for } 0<x<1,
$$

and $v(0)=\lim _{x \rightarrow 0^{+}} v(x)=1 / 2$. Then $\left|a_{2}\right| \leq 1+\lambda v(|a|)$. The result is sharp.

Proof. Let $f \in \mathcal{U}(\lambda)$. Then, we may write (cf. [14])

$$
f^{\prime}(z)\left(\frac{z}{f(z)}\right)^{2}=-z\left(\frac{z}{f(z)}\right)^{\prime}+\frac{z}{f(z)}=1+\lambda \omega(z),
$$

where $\omega: \mathbb{D} \rightarrow \mathbb{D}$ is analytic with $\omega(0)=\omega^{\prime}(0)=0$. By the Schwarz' lemma, we have $|\omega(z)| \leq|z|^{2}$ in $\mathbb{D}$ and hence, $\left|U_{f}(z)\right| \leq|z|^{2}$ for $z \in \mathbb{D}$. In view of $11, f(z)=z+\sum_{n=2}^{\infty} a_{n} z^{n} \in \mathcal{U}(\lambda)$ if and only if

$$
\frac{z}{f(z)}=1-a_{2} z+\lambda z \int_{0}^{z} \omega(t) d t \neq 0, \quad z \in \mathbb{D}
$$

where $\omega \in \mathcal{B}$. By assumption $\omega(0)=a \in \mathbb{D}$. As in the proof of $[19$, Theorem 1], assume on the contrary that

$$
\left|a_{2}\right|=\frac{1+\lambda v(|a|)}{r}, \quad r \in(0,1)
$$


and consider the function $F$ defined by

$$
F(z)=\frac{1}{a_{2}}\left[1+\lambda z \int_{0}^{z} \omega(t) d t\right], \quad z \in \mathbb{D} .
$$

Then, according to the Schwarz-Pick lemma applied to $\omega \in \mathcal{B}$, we can easily obtain that

$$
|\omega(z)| \leq \frac{|a|+|z|}{1+|a z|}, \quad z \in \mathbb{D},
$$

and thus, as in the proof of [19, Theorem 2], it follows that

$$
\left|\int_{0}^{z} \omega(t) d t\right| \leq v(|a|)<1, \quad z \in \mathbb{D}
$$

where $v(x)$ is defined as in the statement. Consequently, for $|z| \leq r$, we get by $(13)$

$$
|F(z)| \leq \frac{1}{\left|a_{2}\right|}\left[1+\lambda|z|\left|\int_{0}^{z} \omega(t) d t\right|\right] \leq \frac{1+r \lambda v(|a|)}{\left|a_{2}\right|}=\frac{(1+r \lambda v(|a|)) r}{1+\lambda v(|a|)}<r
$$

Hence $F$ is a mapping of the closed disk $\overline{\mathbb{D}}_{r}$ into itself, where $\mathbb{D}_{r}=\{z$ : $|z|<r\}$. Secondly, we have for $z_{1}$ and $z_{2}$ in $\overline{\mathbb{D}}_{r}$,

$$
\begin{aligned}
& \left|F\left(z_{1}\right)-F\left(z_{2}\right)\right|=\frac{\lambda r}{1+\lambda v(|a|)}\left|z_{1} \int_{0}^{z_{1}} \omega(t) d t+\left(-z_{1}+z_{1}-z_{2}\right) \int_{0}^{z_{2}} \omega(t) d t\right| \leq \\
& \quad \leq \frac{\lambda r}{1+\lambda v(|a|)}\left(\left|z_{1}\right|\left|\int_{z_{2}}^{z_{1}} \omega(t) d t\right|+\left|z_{1}-z_{2}\right|\left|\int_{0}^{z_{2}} \omega(t) d t\right|\right) \leq \\
& \leq \frac{\lambda r}{1+\lambda v(|a|)}\left(\left|z_{1}\right|+v(|a|)\right)\left|z_{1}-z_{2}\right| \leq \frac{\lambda r(r+v(|a|))}{1+\lambda v(|a|)}\left|z_{1}-z_{2}\right|<r\left|z_{1}-z_{2}\right| .
\end{aligned}
$$

Therefore, $F$ is a contraction of the disk $\overline{\mathbb{D}}_{r}$ and according to Banach's fixed point theorem, $F$ has a fixed point in $\overline{\mathbb{D}}_{r}$. This implies that there exists a $z_{0} \in \mathbb{D}_{r}$ such that $F\left(z_{0}\right)=z_{0}$ which contradicts 12 at $z_{0} \in \mathbb{D}$ 
(and thus, 13$)$ is not true for any $r \in(0,1)$ ). Hence, we must have $\left|a_{2}\right| \leq 1+\lambda v(|a|)$ for $f \in \mathcal{U}(\lambda)$.

To prove that the second coefficient inequality is sharp, we consider

$$
\omega(z)=\frac{z+a}{1+a z}, \quad a \in(0,1)
$$

and we use the fact that $v(a)=\int_{0}^{1} \omega(t) d t$. Hence,

$$
1-(1+\lambda v(a)) z+\lambda z \int_{0}^{z} \omega(t) d t=1-z-\lambda z \int_{z}^{1} \omega(t) d t=: G(z) .
$$

We claim that $G(z) \neq 0$ in $\mathbb{D}$. Since $G(0)=1$, we may assume on the contrary that there exists a $z \in \mathbb{D} \backslash\{0\}$ such that $G(z)=0$. This is equivalent to

$$
\frac{1}{\lambda z}=\frac{1}{1-z} \int_{z}^{1} \omega(t) d t
$$

As

$$
\left|\frac{1}{\lambda z}\right|>1 \text { and }\left|\frac{1}{1-z} \int_{z}^{1} \omega(t) d t\right| \leq 1
$$

we have now proved that $G(z) \neq 0$ for $z \in \mathbb{D}$. In particular, this implies that the function $f$ defined by

$$
f(z)=\frac{z}{1-(1+\lambda v(a)) z+\lambda z \int_{0}^{z} \omega(t) d t}
$$

belongs to the family $\mathcal{U}(\lambda)$, where $\omega$ is given by (14). This proves the sharpness.

Moreover, one can show that a similar sharp inequality is valid for any $\omega$ as above.

Since $\left|\int_{z_{1}}^{z_{2}} \omega(t) d t\right| \leq\left|z_{1}-z_{2}\right|$, the function $\int_{0}^{z} \omega(t) d t$ is uniformly continuous in the open unit disk. Therefore this function can be extended continuously onto the closed unit disk. Hence, the real functional $m(\omega):=$ 
$:=\max \left\{\left|\int_{0}^{z} \omega(t) d t\right|: z \in \overline{\mathbb{D}}\right\}$ is well defined. Suppose that $f \in \mathcal{U}(\lambda)$ is given by

$$
f(z)=\frac{z}{1-a_{2} z+\lambda z \int_{0}^{z} \omega(t) d t}
$$

for some $0 \leq \lambda<1$, where $\omega \in \mathcal{B}$. Then

$$
\left|a_{2}\right| \leq 1+\lambda m(\omega)
$$

is valid and this inequality is sharp.

In order to prove this inequality, we assume again that

$$
\left|a_{2}\right|=\frac{1+\lambda m(\omega)}{r}, \quad r \in(0,1)
$$

and do similar steps as in the proof of Theorem 4. The inequality 15 can be shown to be sharp in the following way: Consider

$$
\tilde{\omega}(z)=e^{i \varphi} \omega\left(e^{i \theta} z\right),
$$

where $\varphi, \theta \in[0,2 \pi)$ are chosen such that

$$
m(\omega)=\int_{0}^{1} \tilde{\omega}(t) d t
$$

Next, we may proceed as before to complete the proof. However, we omit the details to avoid a repetition of the arguments.

A more detailed consideration of these cases can give more explicit bounds for $\left|a_{2}\right|$ as follows.

Theorem 5. Let $f \in \mathcal{U}(\lambda), \lambda \in(0,1)$, have the form 10 for some analytic function $\omega$ such that $|\omega(z)| \leq 1$ and $\omega(0)=a \in \overline{\mathbb{D}}$. Let further

$$
B_{a}(z)=\frac{1}{\bar{a}}-\frac{1-|a|^{2}}{\bar{a}^{2} z} \log (1+\bar{a} z)=a+\left(1-|a|^{2}\right) \sum_{k=1}^{\infty}(-\bar{a})^{k-1} \frac{z^{k}}{k+1}
$$

for $a \in \overline{\mathbb{D}}$. Then $\left|a_{2}\right| \leq 1+\lambda \max \left\{\left|B_{a}\left(e^{i \tau}\right)\right|: \tau \in[0,2 \pi]\right\}$. The inequality is sharp. 
Proof. The function $f$ considered here by 10 is a member of the class $\mathcal{U}(\lambda)$ if and only if $z / f(z) \neq 0$, which is equivalent to

$$
a_{2} \neq \frac{1}{z}+\lambda \int_{0}^{z} \omega(t) d t:=C_{\omega}(z), \quad z \in \mathbb{D} .
$$

Using the above argument, it is clear that the function $C_{\omega}$ can be extended continuously onto the boundary $\partial \mathbb{D}$. Moreover this function is univalent on $\overline{\mathbb{D}}$. The proof of this assertion is similar to the above arguments. Indeed if $C_{\omega}\left(z_{1}\right)=C_{\omega}\left(z_{2}\right)$ for some $z_{1} \neq z_{2}, z_{1}, z_{2} \in \overline{\mathbb{D}}$, then

$$
\frac{\lambda}{z_{1}-z_{2}} \int_{z_{1}}^{z_{2}} \omega(t) d t=\frac{1}{z_{1} z_{2}}
$$

which is not possible. Thus, $C_{\omega}$ is univalent on $\overline{\mathbb{D}}$ and therefore, for each $\omega$, the curve $C_{\omega}\left(e^{i \theta}\right), \theta \in[0,2 \pi]$, is a Jordan curve which divides the plane into two components. Let us call the bounded closed component $\overline{\mathbb{C}} \backslash C_{\omega}(\mathbb{D})=: A_{2}(\omega)$. Obviously, the function $f$ is in the class $\mathcal{U}(\lambda)$ if and only if

$$
a_{2} \in \bigcup_{\omega(0)=a} A_{2}(\omega)
$$

Now, we look at the curves $C_{\omega}\left(e^{i \theta}\right), \theta \in[0,2 \pi]$. Since $\omega(0)=a$, the modulus of the function

$$
\frac{\omega(z)-a}{1-\bar{a} \omega(z)}
$$

is bounded by unity in the unit disk and this function vanishes at the origin. This means that $\omega$ can be represented in the form

$$
\omega(z)=\frac{a+z \varphi(z)}{1+\bar{a} z \varphi(z)},
$$

where $\varphi$ is analytic in $\mathbb{D}$ and $|\varphi(z)| \leq 1$ for $z \in \mathbb{D}$. In other words, $\omega(z)$ is subordinate to $(a+z) /(1+\bar{a} z), z \in \mathbb{D}$. Since the function $(a+z) /(1+\bar{a} z)$ maps the unit disk onto the unit disk, a convex domain, we may now use a theorem proved by Hallenbeck and Ruscheweyh in [11] (compare with [13. Theorem 3.1b]). In our case we use the fact that the function

$$
p(z)=\frac{1}{z} \int_{0}^{z} \omega(t) d t
$$


satisfies the subordination relation

$$
p(z)+z p^{\prime}(z)=\omega(z) \prec \frac{a+z}{1+\bar{a} z}=h(z) .
$$

According to the above theorem, in this case the function $p$ is subordinate to the function

$$
\frac{1}{z} \int_{0}^{z} h(t) d t=\frac{1}{z} \int_{0}^{z} \frac{a+t}{1+\bar{a} t} d t=B_{a}(z)
$$

Therefore, we get the representation

$$
\int_{0}^{z} \omega(t) d t=\frac{1}{\varphi(z)} \int_{0}^{z \varphi(z)} \frac{a+t}{1+\bar{a} t} d t=z B_{a}(z \varphi(z))
$$

where $\varphi$ is analytic in $\mathbb{D}$ and $|\varphi(z)| \leq 1$ for $z \in \mathbb{D}$. Since $B_{a}$ is analytic in the closed unit disk this representation together with the above considerations implies that

$$
\left|a_{2}\right| \leq \sup _{z \in \mathbb{D}, \theta \in[0,2 \pi]}\left|e^{-i \theta}+\lambda e^{i \theta} B_{a}(z)\right| \leq 1+\lambda \max \left\{\left|B_{a}\left(e^{i \tau}\right)\right|: \tau \in[0,2 \pi]\right\} .
$$

Now, we have to prove the sharpness of the inequality. To that end, let $\tau_{0}$ be chosen such that

$$
\left|B_{a}\left(e^{i \tau_{0}}\right)\right|=\max \left\{\left|B_{a}\left(e^{i \tau}\right)\right|: \tau \in[0,2 \pi]\right\} \text {, and } B_{a}\left(e^{i \tau_{0}}\right)=e^{i \alpha}\left|B_{a}\left(e^{i \tau_{0}}\right)\right| .
$$

We take $2 \theta=-\alpha, \psi=\tau_{0}-\theta$, consider the function

$$
\omega(z)=\frac{a+z e^{i \psi}}{1+\bar{a} z e^{i \psi}}
$$

and let $a_{2}=e^{-i \theta}+\lambda e^{i \theta} B_{a}\left(e^{i \tau_{0}}\right)$. Then we have

$$
\left|a_{2}\right|=\left|e^{-2 i \theta}+\lambda e^{i \alpha}\right| B_{a}\left(e^{i \tau_{0}}\right)||=1+\lambda\left|B_{a}\left(e^{i \tau_{0}}\right)\right| .
$$

Further, we consider

$$
D(z)=1-\left(e^{-i \theta}+\lambda e^{i \theta} B_{a}\left(e^{i \tau_{0}}\right)\right) z+\lambda z \int_{0}^{z} \frac{a+t e^{i \psi}}{1+\bar{a} t e^{i \psi}} d t .
$$


It is easily seen that in our case

$$
D(z)=1-\left(e^{-i \theta}+\lambda e^{i \theta} B_{a}\left(e^{i \tau_{0}}\right)\right) z+\lambda z^{2} B_{a}\left(z e^{i \psi}\right) \text { and } D\left(e^{i \theta}\right)=0 .
$$

The assumption that there would exist a second zero $w$ of $D$ in the unit disk, via the equation $D(w)=D\left(e^{i \theta}\right)$ leads to

$$
\frac{1}{w}+\lambda \int_{0}^{w} \omega(t) d t=e^{-i \theta}+\lambda \int_{0}^{e^{i \theta}} \omega(t) d t .
$$

Now we proceed similar to a reasoning above. We conclude that this implies

$$
\frac{1}{\lambda w e^{i \theta}}=\frac{1}{e^{i \theta}-w} \int_{w}^{e^{i \theta}} \omega(t) d t .
$$

But this is impossible, since the left hand side has modulus bigger than 1, whereas the right hand side has modulus less than or equal to 1. Hence, the function $f(z)=z / D(z)$ is a member of the class $\mathcal{U}(\lambda)$.

Acknowledgment. The authors thank the referees for their careful reading of the paper.

\section{References}

[1] Aksentév L. A. Sufficient conditions for univalence of regular functions. Izv. Vysš. Učebn. Zaved. Matematika, 1958, no. 3(4), pp. 3-7. (in Russian)

[2] Aksentév L. A., Avhadiev F. G. A certain class of univalent functions. Izv. Vysš. Učebn. Zaved. Matematika, 1970, no. 10, pp. 12-20. (in Russian)

[3] Avkhadiev F. G., Wirths K.-J. Schwarz-Pick type inequalities. Birkhäuser Verlag, Basel-Boston-Berlin, 2009, 156 pp.

[4] Boas H. P. Julius and Julia: Mastering the art of the Schwarz lemma. Amer. Math. Monthly, 2010, vol. 117, pp. 770-785. DOI:10.4169/000298910X521643

[5] de Branges L. A proof of the Bieberbach conjecture. Acta Math., 1985, vol. 154, pp. 137-152. DOI:10.1007/BF02392821

[6] Clunie J. G. Some remarks on extreme points in function theory. in Aspects of Contemporary Complex Analysis, Proc. NATO Adv. Study Inst., University of Durham, Durham, UK, 1979, Academic Press, London, 1980, pp. $137-146$. 
[7] Duren P. L. Univalent functions. Springer-Verlag, 1983.

[8] Fournier R., Ponnusamy S. A class of locally univalent functions defined by a differential inequality. Complex Var. Elliptic Equ., 2007, vol. 52, no. 1, pp. 1-8. DOI:10.1080/17476930600780149

[9] Friedman B. Two theorems on schlicht functions. Duke Math. J., 1946, vol. 13, pp. 171-177. DOI:10.1215/S0012-7094-46-01317-8

[10] Goodman A. W. Univalent functions. Vols. 1-2, Mariner, Tampa, Florida, 1983.

[11] Hallenbeck D. J., Ruscheweyh. St. Subordination by convex functions. Proc. Amer. Math. Soc., 1975, vol. 52, pp. 191-195. DOI:10.2307/2040127

[12] Jack I. S. Functions starlike and convex of order $\alpha$. J. London Math. Soc., 1971, vol. 3, no. 2, pp. 469-474. DOI:10.1112/jlms/s2-3.3.469

[13] Miller S. S., Mocanu P. T. Differential Subordinations, Theory and Applications. Marcel Dekker, New York, Basel, 2000.

[14] Obradović M., Ponnusamy S. New criteria and distortion theorems for univalent functions. Complex Variables: Theory and Appl., 2001, vol. 44, pp. 173-191. DOI: 10.1080/17476930108815354

[15] Obradović M., Ponnusamy S. Radius properties for subclasses of univalent functions. Analysis, 2005, vol. 25, pp. 183-188. DOI:10.1524/anly.2005.25.3.183

[16] Obradović M., Ponnusamy S. Univalence and starlikeness of certain integral transforms defined by convolution of analytic functions. J. Math. Anal. Appl., 2007, vol. 336, pp. 758-767. DOI:10.1016/j.jmaa.2007.03.020

[17] Obradović M., Ponnusamy S. On certain subclasses of univalent functions and radius properties. Rev. Roumanie Math. Pures Appl., 2009, vol. 54, no. 4, pp. 317-329.

[18] Obradović M., Ponnusamy S., Singh V., Vasundhra P. Univalency, starlikesess and convexity applied to certain classes of rational functions. Analysis, 2002, vol. 22, no. 3, pp. 225-242. DOI:10.1524/anly.2002.22.3.225

[19] Obradović M., Ponnusamy S., Wirths K.-J. Geometric studies on the class $\mathcal{U}(\lambda)$. Bull. Malaysian Math. Sci. Soc., 2016, vol. 39, no. 3, pp. 1259-1284. DOI:10.1007/s40840-015-0263-5

[20] Obradović M., Ponnusamy S., Wirths K.-J. On relations between the classes $\mathcal{S}$ and $\mathcal{U}$. J. Analysis, 2016, vol. 24, pp. 83-93. DOI:10.1007/s41478016-0023-4

[21] Obradović M., Ponnusamy S., Wirths K.-J. Logarithmic coefficients and a coefficient conjecture of univalent functions. Monatsh. Math., 2018, vol. 185, no. 3, pp. 489-501. DOI:10.1007/s00605-017-1024-3 
[22] Ponnusamy S., Wirths K.-J. Elementary considerations for classes of meromorphic univalent functions. Lobachevskii J Math., 2018, vol. 39, no. 5, pp. 712-715. DOI: 10.1134/S1995080218050128

[23] Pommerenke Ch. Univalent functions. Vandenhoeck and Ruprecht, Göttingen, 1975.

[24] Rogosinski W. On the coefficients of subordinate functions. Proc. London Math. Soc., 1943, vol. 48, no. 2, pp. 48-82. DOI: 10.1112/plms/s2-48.1.48

[25] Silverman H. Univalent functions with negative coefficicents. Proc. Amer. Math. Soc., 1975, vol. 51, pp. 109-116.

[26] Vasudevarao A., Yanagihara H. On the growth of analytic functions in the class $\mathcal{U}(\lambda)$. Comput. Methods Funct. Theory, 2013, vol. 13, pp. 613-634. DOI:10.1007/s40315-013-0045-8

Received December 26, $201 \%$.

In revised form, March 10, 2018.

Accepted March 12, 2018.

Published online May 15, 2018.

S. Ponnusamy

Department of Mathematics

Indian Institute of Technology Madras

Chennai-600 036, India

E-mail: samy@iitm.ac.in

K.-J. Wirths

Institut für Analysis und Algebra

TU Braunschweig

38106 Braunschweig, Germany

E-mail: kjwirths@tu-bs.de 